The University of San Francisco

USF Scholarship: a digital repository@ Gleeson Library |

Geschke Center

Psychology

College of Arts and Sciences

1999

\title{
Subjective Culture In Health Interventions
}

Gerardo Marín

University of San Francisco, marin@usfca.edu

Follow this and additional works at: http://repository.usfca.edu/psyc

Part of the Psychology Commons, and the Sociology Commons

\section{Recommended Citation}

Marín, G. (1999). Subjective Culture In Health Interventions. In J. Adamopoulos \& Y. Kashima (eds.). Social Psychology and Cultural Context. Newbury Park CA: Sage.

This Other is brought to you for free and open access by the College of Arts and Sciences at USF Scholarship: a digital repository @ Gleeson Library | Geschke Center. It has been accepted for inclusion in Psychology by an authorized administrator of USF Scholarship: a digital repository @ Gleeson

Library | Geschke Center. For more information, please contact repository@usfca.edu. 


\title{
10
}

\section{SUBJECTIVE CULTURE}

IN HEALTH INTERVENTIONS

\author{
/ 10 \\ Gerardo Marín \\ University of San Francisco
}

A substantial number of recent publications in the area of mental and physical health promotion in the United States have argued for the need to develop prevention efforts that appropriately reflect the cultural characteristics of the targeted ethnic or cultural group (e.g., Cross, Bazron, Dennis, \& Isaacs, 1989; Davis \& Voegtle, 1994; Marín, 1993; Marín et al., 1995; Marín, Amaro, Eisenberg, \& Opava-Stitzer, 1993; Orlandi, 1992; Roberts, 1990). This emphasis on identifying and applying the basic characteristics of a culture in health-promotion activities is probably due in part to the importance assigned to social marketing principles in public health and community change programs (e.g., Andreasen, 1995). The increased awareness of the needs and of the numerical importance of ethnic minority groups in the United States also may have contributed to the attention being given to a group's basic cultural characteristics in the development of healthpromotion materials and interventions. During the 1990 census, the major ethnic groups in the United States (African Americans, American Indians, Asian Americans, and Hispanics) accounted for well over $24 \%$ of the total population of the country. That proportion is expected to increase at a fast pace over the next few years so that by the year 2050, the Bureau of the Census estimates that those four ethnic groups will account for the majority of the population of the country. Those figures obviously require that special

AUTHOR'S NOTE: Portions of this chapter were presented at the 26th Interamerican Congress of Psychology, São Paulo, Brazil, July 1997. 
attention be given to the needs of ethnic groups in the United States by developing interventions that properly recognize their cultural characteristics.

This chapter briefly summarizes the role of a subjective culture analysis in the development of culturally appropriate interventions, with particular attention being given to the promotion of health, although the principles apply equally well to fields such as community change, education, and marketing. The relevance of such an analysis within the framework of cultural appropriateness is examined first, followed by examples derived from major prevention efforts carried out among Hispanics in the United States in the areas of cigarette smoking and alcoholic beverage consumption.

\section{DEFINING CULTURALLY APPROPRIATE INTERVENTIONS}

As mentioned above, various recent publications have argued for the need to develop health-promotion interventions that take into consideration the basic characteristics of the individuals being targeted (e.g., Catalano et al., 1993; Cross et al., 1989; Fisher \& Fisher, 1992; Marín, 1993; Marín et al., 1995; Marín, Amaro, et al., 1993; Muninjaya \& Widarsa, 1993-1994; Orlandi, 1992; Weissberg \& Elias, 1993; Winkleby, Flora, \& Kraemer, 1994). These arguments often have been based on the need to take into consideration the cultural characteristics of the various ethnic groups that are part of multicultural societies such as the United States.

As could be expected from authors writing about an evolving concept, different labels have been used. Some have argued for culturally sensitive interventions (e.g., Bayer, 1994) or culturally tailored programs (Ramirez, MacKellar, \& Gallion, 1988), whereas others have suggested the need for culturally competent interventions (e.g., Cross et al., 1989; Orlandi, 1992; Roberts, 1990) or culturally informed etiological and preventive models (Weiss \& Kleinman, 1988). In this chapter, the term culturally appropriate is preferred over its homologous terms because it can be perceived as being more comprehensive and more closely related to the development of community and behavior-change interventions. For example, culturally appropriate can be described as involving more than being sensitive to cultural differences and nuances, as could be implied in the use of the label culturally sensitive. Furthermore, the term culturally competent is reserved for individuals or interveners who are culturally sensitive and culturally informed and who may be in charge of developing or implementing culturally appropriate interventions. Indeed, as suggested by various authors (e.g., Cross et al., 1989; Davis \& Voegtle, 1994; Orlandi, 1992; Roberts, 1990), culturally competent individuals should be expected to have a number of special abilities that are different from the properties expected of the interventions they have developed or are implementing, such as awareness and feelings of comfortableness with cultural differences, consciousness of the parameters of intercultural dynamics, and having the necessary skills to promote personal adaptation to cultural diversity.

Although a number of authors have acknowledged the need for culturally appropriate interventions (including primary through tertiary prevention efforts), little has been written to describe systematically what is implied in developing such interventions (e.g., 
Marín, 1993; Nobles \& Goddard, 1993). In general, the need for developing culturally appropriate interventions has been based on three basic premises. First is the acknowledgment on the part of researchers and interveners of the fact that culture does indeed influence behavior and that there are important examples of cultural diversity around the world and within nations. This is, of course, an assumption too familiar to crosscultural psychologists and to the readers of this book. Nevertheless, this realization seems to have escaped numerous researchers in other fields.

A second important premise in the definition of culturally appropriate interventions is the assumption derived in part from social marketing (Andreasen, 1995; Rogers, 1983) that suggests that whenever interventions are specifically designed for a given group (culturally appropriate in the case of ethnic groups), they will be not only more easily accepted by members of the group but also more effective (Marín, 1993; Rogers, 1983; Uba, 1992; Varela, 1971; Vega, 1992; Winett, 1995). Evidence has begun to accumulate showing that, indeed, culturally appropriate interventions are producing significant changes in behavior among the individuals being targeted (e.g., Marín \& Pérez-Stable, 1995; Pérez-Stable, VanOss Marín, \& Marín, 1993; VanOss Marín, Marín, Pérez-Stable, \& Hauck, 1994).

The third premise is the belief that the development of culturally appropriate interventions must go beyond the adaptation and/or translation of interventions previously developed for members of other ethnic groups (Bayer, 1994; Marín, 1993). This premise does not imply that "mainstream" interventions will not be effective with members of defined cultural groups. Studies have shown, for example, that well-developed "mainstream" prevention interventions are also effective with members of specific ethnic groups (e.g., Sussman et al., 1993) and that certain adapted or modified "mainstream" interventions also can produce some of the desired effects (e.g., Bohon, Santos, SanchezSosa, \& Singer, 1994; Botvin, Schinke, Epstein, \& Diaz, 1994; Botvin, Schinke, Epstein, Diaz, \& Botvin, 1995). Rather, this assumption argues that the level of acceptability and effectiveness of a prevention intervention may be higher when the intervention is designed to meet the characteristics and guidelines of a culturally appropriate intervention that are mentioned below. As a matter of fact, certain authors (e.g., Reid, Killoran, McNeill, $\&$ Chambers, 1992) have argued that acceptability of an intervention should be one of the central criteria when choosing health-promotion interventions.

One recent effort at defining the components of culturally appropriate interventions (Marín, 1993) has been heavily influenced by the work of Harry Triandis and indeed incorporates a limited analysis of the subjective culture of a group as part of the process. This approach to developing culturally appropriate interventions suggests incorporating three basic components: (a) the basic cultural values or cultural dimensions of the targeted group, (b) certain behavior-specific aspects of the subjective culture of the group (e.g., attitudes, expectancies, situational antecedents, and perceived norms), and (c) groupspecific preferences for intervention modalities (e.g., preferred sources and/or channels of information, valued behavioral activities, etc.). As mentioned above, one basic assumption of this model for culturally appropriate interventions is the belief that there are important differences across cultures and ethnic groups to warrant the development of group-specific interventions. 


\section{BASIC COMPONENTS OF A CULTURALLY APPROPRIATE INTERVENTION}

The paragraphs that follow describe what is meant by each of the components of a culturally appropriate intervention (Marín, 1993) and provide examples derived from our work with Hispanics to illustrate not only cultural differences but also how each component helps to shape a culturally appropriate intervention in terms of its content and strategies.

\section{Group-Specific Cultural Dimensions or Syndromes}

The model for developing culturally appropriate interventions proposed by Marín (1993) suggests that the intervention needs to be informed by the basic cultural dimensions or cultural syndromes that characterize individuals of the targeted group. In the case of Hispanics and Latin Americans, there is substantial research providing support for the existence of culture-specific cultural dimensions and scripts. For example, work by Triandis and colleagues (Marín \& Triandis, 1985; Triandis, 1990; Triandis, Marín, Lisansky, \& Betancourt, 1984) has shown the significance of a collectivist orientation among Hispanics that supports the influence of members of the collective in shaping an individual's behavior as well as the importance of the role of family members as behavioral and attitudinal referents and providers of emotional, economic, and other types of support (Sabogal, Marín, Otero-Sabogal, VanOss Marín, \& Pérez-Stable, 1987). Indeed, cultural dimensions, when supported by empirical evidence, can serve an important role in the development of culturally appropriate interventions by directing interveners in choosing the overall orientation of the intervention, identifying the role of particular normative members of the community, describing the way in which attitudes and behaviors are changed, and pointing out those beliefs that are central to a group's worldview.

Earlier research in the social sciences had shown the existence of various cultural dimensions among Hispanics, including an orientation toward subjugation to nature, with the inherent sense of powerlessness of human beings toward the forces of nature and toward God's will (Heller, 1966; Kluckhohn \& Strodtbeck, 1961). Nevertheless, some of these early cultural dimensions have received little empirical support probably because they were not well-defined or because they may represent the experiences of rural Hispanics, who no longer represent the majority of Hispanics in the United States. For example, the preference for a subjugation-to-nature perspective has been supported by empirical studies with relatively small samples (Sjostrom, 1988; Szapocznik, Kurtines, \& Hanna, 1979; Szapocznik, Scopetta, Aranalde, \& Kurtines, 1978), whereas other studies have found the opposite (Grebler, Moore, \& Guzman, 1970).

Other cultural dimensions and associated scripts or behavioral patterns that are commonly assigned to Hispanics seem to have found stronger support in the literature. For example, the cultural syndrome of collectivism has, thanks to the pioneering work of Harry Triandis (1990, 1995b, 1996; Kim, Triandis, Kagitcibasi, Choi, \& Yoon, 1994), surfaced as a central cultural dimension of Hispanics. Applications of collectivism in the development of culturally appropriate interventions have been based on shaping an 
intervention that recognizes, supports, enriches, and makes use of important characteristics of collectivist individuals such as the greater valuing of the views and needs of the members of the in-group, the willingness to share resources without concern for individual utilitarian considerations, the acceptance of the group's norms, and personal values such as intrdependence, cooperation, and sociability (Hofstede, 1980; Hui \& Triandis, 1986; Triandis, 1990, 1994a, 1996).

The early work of anthropologists suggested a preference for a being orientation among Hispanics (Heller, 1966; Kluckhohn \& Strodtbeck, 1961) that has received some empirical support and has been of importance in the development of culturally appropriate interventions. This being orientation is primarily based on the importance assigned to the individual and to personal experiences of reality and the generalized concern for human values and the spirit (Bañuelas, 1995; Fitzpatrick, 1971; Goizueta, 1995; Isasi-Díaz \& Tarango, 1992; Magaffey \& Barnett, 1962). This orientation may have been influenced by the Spanish traditions of courteous and congenial demeanor (De Miguel, 1990; Gillin, 1965; Gilmore, 1987; Hooper, 1986; Wagley, 1968; Wolf, 1956) or by the French sense of politesse, or civilized courteous politeness (Montandon, 1995; Picard, 1995). This dimension is seen by various researchers as a possible source of such social scripts as Simpatía (Triandis, Marín, Lisansky, \& Betancourt, 1984), Personalismo, and Respeto that have been central to the design of culturally appropriate interventions targeting Hispanics.

Together with some cultural dimensions, a number of cultural scripts also have been identified as central to Hispanic culture and of importance in the design of culturally appropriate interventions. For example, familialism (also labeled familism by some authors) has been suggested as central to understanding Hispanic culture because it places the family as the main institution in the social world of Hispanics with obligations including solidarity, reciprocity, commitment, nurturance, and loyalty (Kagan, 1977; Triandis, Marín, Hui, Lisansky, \& Ottati, 1984). The familialism script also makes it possible for individuals to benefit from their participation in the familial relationship, including receiving emotional and financial support (Keefe \& Padilla, 1987; Mannino \& Shore, 1976; Murillo, 1976) and protection from physical and emotional stressors (De la Rosa, 1988; Grebler et al., 1970; Markides, Costley, \& Rodriguez, 1981; Markides \& Krause, 1985).

Other important Hispanic cultural scripts that have received empirical support and that can be of importance in the design of culturally appropriate interventions include Simpatia, with its expectation of harmony, loyalty, dignity, friendliness, politeness, affection, respect, and positivity in interpersonal relations (Triandis, Marín, Lisansky, \& Betancourt, 1984), and Personalismo, with its emphasis on individualized and deferential personal interactions (Fitzpatrick, 1971; Padilla, 1964; Wagenheim, 1972).

The model for developing culturally appropriate interventions proposed by Marín (1993) requires not only that interveners and intervention designers need to be aware of those cultural dimensions that show promise in characterizing a cultural groupHispanics in this case- but also that the interventions incorporate components that are in agreement with the dimensions and scripts. For example, and as mentioned below, the cultural dimension of collectivism was useful in shaping a self-help smoking-cessation campaign directed at Hispanics (Marín \& Pérez-Stable, 1995; Pérez-Stable et al., 1993). In this intervention, the opinions of community members were used to support each of the 
various behavior changes promoted by the program, through the use of pictorial vignettes and sidebars with printed testimonials included in a self-help manual as well as through radio and television programming. Likewise, our research has shown that familialism and the associated heightened concern and sense of responsibility for the welfare of family members have been of particular importance among Hispanics in curtailing cigarette smoking and the consumption of alcoholic beverages (e.g., Marín, in press-b; Marín, VanOss Marín, Otero-Sabogal, Sabogal, \& Pérez-Stable, 1989; VanOss Marín, Marín, Pérez-Stable, Otero-Sabogal, \& Sabogal, 1990; VanOss Marín, Pérez-Stable, Marín, Sabogal, \& Otero-Sabogal, 1990).

\section{An Analysis of a Group's Subjective Culture}

A second characteristic of a culturally appropriate intervention according to the model developed by Marin (1993) is the inclusion of the results of an analysis of the subjective culture of a specific group regarding the targeted behavior. This aspect of developing a culturally appropriate intervention involves identifying all or most of the components of a group's subjective culture (e.g., values, norms, attitudes, expectancies) to properly shape the actual content of the intervention materials. In this sense, if research shows that members of a given ethnic group (e.g., Hispanics in the United States) have attitudes toward a targeted behavior (e.g., cigarette smoking) that are different from those held by members of another group (e.g., non-Hispanic whites), then the intervention needs to be shaped so that the attitudes of the targeted group are reflected in the contents of the intervention materials. This is a situation that is particularly important in multicultural societies in which interventions may be shaped by members of one ethnic group (usually those in the numerical majority) and therefore reflect the attitudes of a group that may bear little resemblance to those of the targeted group.

The need for this component of a culturally appropriate intervention is based on the fact that research has shown that members of ethnic and cultural groups share some group-specific attitudes, norms, expectancies, and values related to certain behaviors, which in turn are different from those held by other cultural groups or by members of the numerical majority in multicultural societies. For example, our research (as detailed below) has identified a number of group-specific components of the subjective culture of Hispanics in the United States regarding tobacco smoking (Marín et al., 1989; Marín, VanOss Marín, Pérez-Stable, Sabogal, \& Otero-Sabogal, 1990b; VanOss Marín, Marín, et al., 1990) and the drinking of alcoholic beverages (Marín, in press-b; Marín, Posner, \& Kinyon, 1993), which are significantly different from those held by non-Hispanic whites also residing in the United States. These differences in attitudes, expectancies, norms, and values imply that a culturally appropriate intervention for Hispanics that incorporates the group-specific components of a subjective culture will necessarily be better received and more effective than an intervention that has been developed by non-Hispanic whites reflecting non-Hispanic white attitudes, norms, values, and expectancies.

Indeed, research outside the cross-cultural environment has shown that, when the content of a message is shaped to match the values and needs of the audience, its effectiveness is enhanced (Clary, Snyder, Ridge, Miene, \& Haugen, 1994). In addition, 
researchers (e.g., Catalano et al., 1993) have argued for the need to tailor prevention messages to address group-specific risk behaviors. For example, Catalano et al. (1993) suggest the need to develop drug-abuse-prevention programs for African American youth that take into consideration such risk factors as aggressiveness (which is a prominent risk factor primarily among African Americans) as well as those risk factors that seem to be common to African American and non-Hispanic white youth (e.g., access to marijuana, presence of friends who drink, and lack of out-of-school involvement opportunities).

As mentioned above, evidence has begun to accumulate that supports the notion that there are group-specific attitudes, norms, values, and expectancies for a number of behaviors. Our recent research has shown that Hispanics in the United States have a number of expectancies toward various behaviors that differentiate them from those held by non-Hispanic whites. For example, one of our studies on the expectancies held by Hispanics regarding the consumption of alcoholic beverages (Marín, Posner, \& Kinyon, 1993) showed that Hispanics were more likely to agree with most of the possible expectancies for the consumption of alcoholic beverages (e.g., laughing more, becoming more talkative and more aggressive, being careless, feeling happier, becoming sleepy, and feeling romantic) than were non-Hispanic whites. A more recent study (Marín, in press-b) with large random samples of Mexican Americans in California and Texas again showed that Mexican Americans hold different cognitions than those held by non-Hispanic whites. In this last study, Mexican Americans were more likely to hold a number of negative expectations such as drinking producing fights, violence and aggressiveness, losing self-control, showing a bad example to children, having problems at work and at home, and becoming depressed and careless. In addition, Mexican Americans felt that drinking would make a person more independent and more romantic.

Some of our earlier research has shown that Hispanics also share expectancies regarding cigarette smoking that are quite different from those held by non-Hispanic whites. For example, a series of studies showed that Hispanic smokers are more concerned than non-Hispanic white smokers that, by smoking, they provide a bad example to their children and that they are threatening the good health of children by exposing them to environmental tobacco smoke (Marín et al., 1989; Marín et al., 1990b; VanOss Marín, Marín, et al., 1990). Our research also showed that there were a number of consequent expectancies that more readily discriminated Hispanic from non-Hispanic white smokers. For example, an analysis of expectations about the consequences of cigarette smoking showed the significant concern on the part of Hispanic smokers for damaging the health of children by exposing them to environmental cigarette smoke, whereas non-Hispanic whites were more concerned about the lack of personal control implied in cigarette smoking (Marín et al., 1990b). Hispanic smokers were more readily willing to consider quitting to provide a good example to their children, to improve relationships with their relatives, to breathe more easily, and to have a better taste in their mouths. On the other hand, non-Hispanic white smokers felt that an important consequent expectancy of quitting smoking was the ability to accomplish something difficult (Marin et al., 1990b).

As could be expected, there are expectancies held by Hispanics for behaviors such as the drinking of alcoholic beverages or cigarette smoking that are similar to those held by 
non-Hispanic whites. Likewise, certain demographic variables (e.g., gender, acculturation) and the actual experience with the behavior (e.g., frequency, intensity) also affect the type of expectancies held by Hispanics and non-Hispanic whites (Cervantes, Gilbert, Snyder, \& Padilla, 1990-1991; Marín, in press-b; Marín, Posner, \& Kinyon, 1993; Marín et al., 1989; Marín et al., 1990b). For example, the Marín, Posner, and Kinyon (1993) study found differences due to drinking status (with abstainers being more likely to expect impairment), and the same was true of the study with Mexican Americans (Marín, in press-b). An analysis of antecedent expectations to cigarette smoking showed that Hispanics considered their cigarette smoking as less frequently motivated by events related to relaxation at home or while enjoying a meal than did non-Hispanic whites, whereas both groups considered emotional and social events as important antecedents to smoking (Marín et al., 1990b). Nevertheless, research seems to be fairly consistent in supporting the notion of the existence of group-specific attitudes, norms, expectancies, and values. These differences as well as the similarities across ethnic and cultural groups need to be incorporated into the design of culturally appropriate interventions.

As an example of this integration of subjective culture components with the contents of an intervention, consider the previously mentioned result of our studies in which Hispanic smokers were found to consider the protection of a child's health as a good reason for quitting smoking (e.g., VanOss Marín, Marín, et al., 1990). Likewise, our subjective culture studies showed that preventing the exhibition of a bad example to children was also an important motivator to quit. Our culturally appropriate community intervention directed at Hispanic smokers (Marín \& Pérez-Stable, 1995; Pérez-Stable et al., 1993) made use of those group-specific attitudes (which could also be perceived as reflecting the cultural script of familialism) in a number of ways. Smokers were frequently told in our self-help manual as well as in other media that by quitting smoking they would be protecting the health of their children and not providing a bad example for minors. For example, the first page in the self-help manual (Institutos Nacionales de la Salud, 1993) dealt with answers to the question "Why should I quit smoking?" Four areas (identified as part of the subjective culture study) were covered: (a) to protect the health and welfare of my family; (b) to improve my health; (c) to avoid serious health problems in the future; and (d) to improve my physical appearance. Among the specific reasons mentioned under the first area (protecting the health and welfare of the family), smokers were told that by quitting smoking they would provide a good example to children, prevent illnesses such as emphysema and colds in their children, improve their relationships with relatives, save money, and live many more years to enjoy their children and grandchildren. These expectancies were precisely the ones that had been found to be important to Hispanic smokers during a subjective culture study, and many of them were found to be more important to Hispanic than to non-Hispanic white smokers (Marín et al., 1989, 1990b; VanOss Marín, Marín, et al., 1990).

The same themes related to familialism and the concern for children were carried out in pictures, posters, billboards, and other media that were part of our culturally appropriate smoking-cessation intervention (Marín \& Pérez-Stable, 1995; Pérez-Stable et al., 1993). For example, a poster showed a young child facing a large floor mirror while dressed in his father's clothes and holding a cigarette in his hand. Billboards often 
proclaimed "Deje de fumar por usted y por su familia" (Quit smoking for you and for your family). The state of California, in its smoking-cessation campaign targeting Hispanics, also made use of these subjective culture findings by producing billboards depicting a burning cigarette on an ashtray placed in front of the portrait of a young girl with the caption "Si tu fumas, ella fuma" (If you smoke, she smokes).

What these examples show is that the results of the subjective culture study of a specific cultural group regarding a behavior can be effectively used to develop the messages that make up the intervention. In many cases, the basic motivation or orienting principles of the intervention will differ significantly from those developed for other groups given the differences in attitudes, expectancies, values, and norms identified in the subjective culture study. As mentioned above, our smoking-cessation intervention with Hispanics had as a primary motivator the protection of children (a value concordant with collectivism and with familialism). This is a principle fairly different from smokingcessation programs developed by and oriented toward non-Hispanic whites in which gaining personal control over one's life (an individualist concern) seems to be a central motivation for quitting smoking.

\section{Group-Specific Preferences for Intervention Modalities}

The third component of the model of a culturally appropriate intervention as suggested by Marin (1993) is the identification and use of group-specific preferences for intervention modalities. This suggestion is based on the assumption that cultures may have specific preferences (based on perceived usefulness, credibility, trustworthiness, motivating power, etc.) for certain behavior-change modalities (e.g., individualized counseling vs. group-based approaches and information disseminated through electronic media vs. printed media). Likewise, within a given choice of behavior-change modality, the assumption can be made that cultures differ in their evaluation (again based on characteristics such as perceived credibility, trustworthiness, and motivating power) of various sources of information (e.g., physicians, priests, peers, teachers, actors, and entertainers) as well as of various channels for the dissemination of information (e.g., television, radio, books, newspapers, and magazines).

The model for developing a culturally appropriate intervention that we have suggested (Marín, 1993) proposes that an evaluation needs to be made of these cultural preferences and that only those with marked positive characteristics should be used. Indeed, communication theoreticians have often argued for the use of appropriate sources and channels of information in developing effective messages and interventions (Bettinghaus, 1986), and evidence exists that shows culture- or group-specific differences in the evaluations of various intervention strategies (Marin, 1996; Marín \& VanOss Marín, 1990) and in the patterns of use of various information dissemination channels (Alcalay et al., 1987-1988; Marín, in press-a; Marín \& Gamba, in press).

Currently, there is evidence showing that members of certain cultural or ethnic groups have little interest or show high dropout rates or low participation rates in some intervention strategies that may not fit their needs or that may conflict with cherished cultural beliefs. This has been shown for self-help groups in which participation is low 
and dropout rates are fairly high (Ahluwalia \& McNagny, 1993; Pérez-Stable et al., 1993). An increasing body of knowledge also shows culture-specific patterns of use of certain media. For example, Hispanics prefer certain approaches or types of media in proportions that are different from those of non-Hispanic whites or of other ethnic groups in the United States. Some of our research (Alcalay et al., 1987-1988) showed that Hispanics in San Francisco spend approximately the same amount of time per day watching television (approximately 3 hours) as they spend listening to the radio, although a greater proportion watch Spanish-language television (72\%) than listen to Spanish-language radio $(47 \%)$. This same survey showed that relatively few respondents $(60 \%)$ read newspapers on a weekly basis. National data indeed show a strong preference by Hispanics of electronic media over printed media. For example, a 1992 survey of Hispanic households (cited in Reddy, 1995) showed that a larger proportion mentioned exposure to television $(95 \%)$ and to radio (84\%) than to newspapers (66\%) and magazines (40\%). Though these figures may reflect actual availability of certain media in a given market, they need to be considered in deciding the specific channel to be used in developing an intervention. In the case of Hispanics, the above-mentioned data would suggest that special attention needs to be given to the use of electronic media (radio and television).

A few recent studies have analyzed the evaluation made by Hispanics of various channels of information (Marín, 1996; Marín \& VanOss Marín, 1990). In our earlier study, we found that Hispanics rated an AIDS hotline as the most credible source of information about AIDS or HIV infection, followed by printed channels (e.g., books, pamphlets, and newspaper articles) and television and radio commercials (Marín \& VanOss Marín, 1990). More recent studies analyzed various channels of information for tobacco and alcohol information in terms of their credibility and motivating power among large random samples of Hispanics and non-Hispanic whites in California and Texas. In those studies (Marín, 1996), we found that printed channels (books and newspaper and magazine articles) and television news programs were evaluated as the most credible and motivating by Hispanics and non-Hispanic whites alike. Television and radio commercials were generally next in credibility, followed by display media such as posters, billboards, and bus signs. The least-credible channels were serialized soaps on radio or television. In general, Hispanics tended to assign greater credibility to the various channels than did non-Hispanic whites. The more-acculturated Hispanics were also found to rate the various channels in a way more similar to that of non-Hispanic whites than to that of the less-acculturated Hispanics.

The data on channel characteristics tend to suggest that attention should be given to electronic media in developing community interventions, given the high esteem in which they are held by Hispanics and non-Hispanic whites alike. This is therefore an area in which cultural differences are of less importance than in other areas of the model for culturally appropriate interventions developed by Marin (1993). Nevertheless, the data are also useful in suggesting that certain channels that have been considered to be "culturally relevant" for Hispanics (such as serialized soaps-telenovelas and fotonovelas) because of their saliency in Latin America may owe their popularity to their entertainment value, but they are perceived as having low (although not insignificant) value as sources of information about health. 
Various health-promotion programs and interventions have emphasized the involvement of individuals perceived as highly credible and expert. For example, health care providers, and particularly physicians, have been proposed as effective sources of information because of their credibility, trustworthiness, and perceived expertise (Reid et al., 1992); other programs have suggested the use of priests and pastors because of their central role in shaping norms among certain ethnic and cultural groups (Stillman, Bone, Rand, Levine, \& Becker, 1993). This is an area in which empirical information needs to be obtained to use those sources of information who are perceived by members of the targeted group as experts, highly credible, trustworthy, and motivating. Data from our studies on alcohol and tobacco (Marín, 1996) information dissemination are instructive here.

Our surveys (Marín, 1996) with large random samples of Hispanics and nonHispanic whites in California and Texas showed important differences in the ways in which various sources of information are perceived across cultural groups. In general, Hispanics perceived the following sources as most credible for transmitting tobacco- or alcohol-related information: physicians, cancer patients or former alcoholics, peers, friends, and priests. Non-Hispanic whites reported similar sources as the most credible, except that teachers replaced priests as one of the five most credible sources. The data showed that Hispanics tended to rate most sources in a more positive fashion than did non-Hispanic whites in terms of credibility, expertise, and trustworthiness. Likewise, these studies showed important differences among Hispanics as a result of their level of acculturation. The most highly acculturated respondents tended to rate the various sources of information in a fashion more similar to that of non-Hispanic whites than to that of the less-acculturated Hispanics.

The data on the evaluation of sources of information provide support for certain levels of generalizability across the two ethnic groups included in our studies while at the same time point to the particularly high possible impact of priests as disseminators of health information among Hispanics. What these data also show is that individuals evaluated sources and channels of information differentially and that a properly targeted culturally appropriate intervention needs to identify and use those sources and channels of information that are rated most highly by members of the group or groups in question. Interventions can be expected to be better received and more effective when they make use of highly credible and trusted sources and channels of information. Indeed, that has been our experience with the smoking-cessation community intervention that targeted Hispanics (Marín \& Pérez-Stable, 1995; Pérez-Stable et al., 1993). Physicians and individuals who could be construed to be members of the community were used in the self-help manual and in television and radio commercials as sources of information on reasons for quitting smoking; they shared their experiences of successfully quitting smoking as well.

\section{CONCLUDING REMARKS}

The model for a culturally appropriate intervention proposed by Marín (1993) was derived from our experiences developing and evaluating a smoking-cessation community program for Hispanics in San Francisco, California, for approximately 6 years. The 
intervention is described in a number of publications (Marín \& Pérez-Stable, 1995; Marín, VanOss Marín, Pérez-Stable, Sabogal, \& Otero-Sabogal, 1990a; Pérez-Stable et al., 1993; VanOss Marín et al., 1994) and included wide distribution of the self-help manual mentioned above (more than 70,000 copies were distributed); the production of television and radio commercials and talk shows; the provision of individualized counseling over the telephone on approaches to quitting cigarette smoking; the implementation of a communitywide raffle for those quitting smoking within a given period of time; and the printing and distribution of informational fliers, posters, bumper stickers, and bus signs.

Evaluations of the smoking-cessation community intervention (Marín et al., 1990a; Marín \& Pérez-Stable, 1995; Pérez-Stable et al., 1993; VanOss Marín et al., 1994) as well as of the self-help manual (Pérez-Stable, Sabogal, Marín, VanOss Marín, \& Otero-Sabogal, 1991) showed that the shaping of an intervention using the group-specific characteristics (norms, expectancies, attitudes, etc.) identified through a subjective culture study produced important cognitive and behavioral changes. Our data showed that, after approximately 6 years of implementation of the culturally appropriate intervention, Hispanics in San Francisco (the targeted group) reported great acceptance of the intervention and its messages, including an increased awareness of the intervention's components, increased levels of information about the effects of cigarette smoking, a decrease in the number of cigarettes smoked, and an increase in the number of attempts at quitting.

As mentioned at the beginning, this chapter was designed to describe the role of culture and its analysis in the design of culturally appropriate interventions. As societies become more multicultural, the need for such interventions will necessarily increase as we try to develop optimally effective and efficient interventions to promote health as well as to introduce other much-needed changes in society. The psychological study of culture and its determinants will necessarily help interveners, social agents, and community activists to design better and more appropriate interventions. The techniques and approaches used by cross-cultural researchers, many of them pioneered by Harry Triandis, will significantly contribute not only to the development of better interventions but also to the enhancement of our understanding of human behavior and to the improvement of the human condition-certainly, a noteworthy contribution. 